\title{
Antinociceptive effects of oleuropein in experimental models of neuropathic pain in male rats
}

\author{
Huayong Chen ${ }^{1, *}$, Dandan Ma ${ }^{1, *}$, Huapeng Zhang ${ }^{1}$, Yanhong Tang ${ }^{2}$, Jun Wang ${ }^{3}$, Renhu $\mathrm{Li}^{4}$, Wen Wen ${ }^{5}$, and \\ Yi Zhang ${ }^{6}$
}

'Department of Anesthesiology, Yidu Central Hospital of Weifang, Weifang, Shandong, China

${ }^{2}$ Department of Anesthesiology, Hospital T.C.M Affiliated to Southwest Medical University, Luzhou, Sichuan, China

${ }^{3}$ Orbital Disease and Ophthalmoplasty, Department of Ophthalmological Hospital, The Second Hospital of Jilin, Changchun, Jilin, China

${ }^{4}$ Department of Anesthesiology, The Lu'an Affiliated Hospital of Anhui Medical University, Lu'an, Anhui Province, China

${ }^{5}$ Department of Anesthesiology, Affiliated Hospital of Guilin Medical University, Guilin, Guangxi, China

${ }^{6}$ Department of Anesthesiology, Tongji Hospital Affiliated Tongji Medical College, Huazhong Science and Technology University, Wuhan, Hubei, China

Received September 24, 2020

Revised November 16, 2020

Accepted November 17, 2020

Handling Editor: Jong Yeon Park

\section{Correspondence}

Yi Zhang

Department of Anesthesiology, Tongji Hospital Affiliated Tongji Medical College, Huazhong Science and Technology University, Wuhan, Hubei 430030, China Tel: +86-139-86039131

Fax: +86-139-86039131

E-mail: doc_zhang18@sina.com

*These authors contributed equally to this work.
Background: The present investigation explored the therapeutic actions of oleuropein along with the possible signaling pathway involved in attenuating neuropathic pain in chronic constriction injury (CCl) and vincristine-induced neuropathic pain in male rats.

Methods: Four loose ligatures were placed around the sciatic nerve to induce $\mathrm{CCl}$, and vincristine $(50 \mu \mathrm{g} / \mathrm{kg})$ was injected for 10 days to develop neuropathic pain. The development of cold allodynia, mechanical allodynia, and mechanical hyperalgesia was assessed using different pain-related behavioral tests. The levels of $\mathrm{H}_{2} \mathrm{~S}$, cystathionine- $\gamma$-lyase (CSE), cystathionine- $\beta$-synthase (CBS), orexin, and nuclear factor erythroid-2-related factor 2 (Nrf2) were measured in the sciatic nerve.

Results: Treatment with oleuropein for 14 days led to significant amelioration of behavioral manifestations of neuropathic pain in two pain models. Moreover, oleuropein restored both $\mathrm{CCl}$ and vincristine-induced decreases in $\mathrm{H}_{2} \mathrm{~S}, \mathrm{CSE}, \mathrm{CBS}$, orexin, and Nrf2 levels. Co-administration of suvorexant, an orexin receptor antagonist, significantly counteracted the pain-attenuating actions of oleuropein and Nrf2 levels without modulating $\mathrm{H}_{2} \mathrm{~S}$, CSE and CBS.

Conclusions: Oleuropein has therapeutic potential to attenuate the pain manifestations in $\mathrm{CCl}$ and vincristine-induced neuropathic pain, possibly by restoring the CSE, $\mathrm{CBS}$, and $\mathrm{H}_{2} \mathrm{~S}$, which may subsequently increase the expression of orexin and Nrf2 to ameliorate behavioral manifestations of pain.

Key Words: Hydrogen Sulfide; Hyperalgesia; Neuralgia; Neuropeptides; NF-E2Related Factor 2; Nociception; Orexins; Orexin Receptor Antagonists; Polyphenols; Vincristine.

\section{INTRODUCTION}

Injury to the peripheral nerves or in the central nervous system is responsible for the induction of neuropathic pain, which is characterized by allodynia and hyperalgesia. The presence of pain symptoms despite the healing of (c) This is an open-access article distributed under the terms of the Creative Commons Attribution Non-Commercial License (http://creativecommons.org/licenses/by-nc/4.0/), which permits unrestricted non-commercial use, distribution, and reproduction in any medium, provided the original work is properly cited.

(C) The Korean Pain Society, 2021
Author contributions: Huayong Chen: Investigation; Dandan Ma: Investigation; Huapeng Zhang: Investigation; Yanhong Tang: Formal analysis; Jun Wang: Writing/manuscript preparation; Renhu Li: Writing/manuscript preparation; Wen Wen: Writing/manuscript preparation; Yi Zhang: Supervision. 
the injury, and being non-responsive to analgesics are the other characteristic features of neuropathic pain [1]. There have been drugs approved to symptomatically manage neuropathic pain; however, the therapeutic management of these patients have still not been optimized. Oleuropein is a polyphenolic compound, and is the main active constituent of olive oil [2]. Research studies have shown the therapeutic potential of oleuropein in several diseases, including depression, Parkinson's disease, cancer, ischemiareperfusion injury, metabolic disorders, etc. [2-4]. Apart from these, the inhibitory actions of oleuropein containing leaf extract of Ligustrum vulgare, in diabetic neuropathic pain, have been documented [5]. Moreover, Olea europaea-derived oleuropein is shown to exhibit antiinflammatory and anti-nociceptive actions [6] and prevent anti-nociceptive tolerance against morphine [7].

$\mathrm{H}_{2} \mathrm{~S}$ is a gaseous neurotransmitter with diverse physiological as well as pathophysiological functions $[8,9]$. Cystathionine- $\gamma$-lyase (CSE) and cystathionine- $\beta$-synthase (CBS) synthesize $\mathrm{H}_{2} \mathrm{~S}$ in the body [10], which may impart useful effects in attenuating neuropathic pain in different experimental models of pain including chronic constriction injury (CCI) $[11,12]$ and the oxaliplatin-induced pain model [13]. Orexins are neuropeptides that play a crucial role in sleep, wakefulness, and appetite [14]. Apart from these, studies have shown that orexins may exert analgesic actions in neuropathic pain models, including CCI [15], oxaliplatin [16], and diabetic pain models [17]. The protective role of $\mathrm{H}_{2} \mathrm{~S}[11,12]$ and orexins [15] in neuropathic pain models have been mainly attributed to their actions on the neurons of the dorsal horn and spinal cord. Nuclear factor erythroid-2-related factor 2 (Nrf2), a transcription factor, is very important in maintaining the levels of antioxidants in the body [18]. The decrease in the levels of Nrf2 is associated with the development of neuropathic pain [12].

There have been studies showing that polyphenols may produce their beneficial effects by increasing the levels of $\mathrm{H}_{2} \mathrm{~S}[19,20]$, orexin [21], and Nrf2 [22]. Moreover, there are direct studies showing that oleuropein (polyphenol) increases the expression of Nrf2 [23]. Considering the association of polyphenols with $\mathrm{H}_{2} \mathrm{~S}$, orexin, and $\mathrm{Nrf2}$, it was hypothesized that oleuropein (polyphenol) may also affect these biochemical molecules to modulate pain. Therefore, the current study was designed to explore the therapeutic usefulness of oleuropein in CCI and vincristine-induced neuropathic pain. Moreover, the possible role of $\mathrm{H}_{2} \mathrm{~S}, \mathrm{CSE}$, CBS, orexin, and Nrf2 was also explored in oleuropeinmediated analgesic actions in male rats.

\section{MATERIALS AND METHODS}

\section{Animals, drugs, and chemicals}

For this study, male Wistar rats (200-250 g) were used. The Institutional Animals Ethics Committee of Yidu Central Hospital of Weifang approved the experimental protocol (approval number: 201900876B097). Suvorexant was purchased from AdooQ BioScience, Irvine, CA; oleuropein and dimethyl sulfoxide (DMSO) were purchased from Sigma-Aldrich, St. Louis, MO. The doses of suvorexant (10 and $20 \mathrm{mg} / \mathrm{kg}$ ) [24] and oleuropein (10 and $20 \mathrm{mg} / \mathrm{kg}$ ) [25] were selected on the basis of previously published literature. Suvorexant was dissolved in $10 \%$ DMSO, and oleuropein was dissolved in water. Vincristine sulfate was dissolved in normal saline. The drugs oleuropein and suvorexant were administered to CCI, vincristine, and non-treated rats daily, by the oral route. The oral route was chosen to avoid the pain induction that may arise due to a daily injection of drugs. Since the direct administration of drugs by the oral route may also produce stress, drugs were administered by dissolving them in drinking water. To avoid variation in drinking water, drugs were dissolved in a specific volume of drinking water ( $200 \mathrm{~mL}$ of), and care was taken so that animals consumed the entire $200 \mathrm{~mL}$ of water.

\section{CCI model}

Thiopental sodium (40 mg/kg intraperitoneal [i.p.]) was used to anesthetize rats and the sciatic nerve was loosely ligated at four places with a gap of $1 \mathrm{~mm}$, using silk 4-0 thread. Afterward, suturing was performed to cover the muscle and skin. The pain-related behavioral parameters were performed on the day before surgery and on the 14th day after surgery [26]. Amongst the different animal models, the CCI model is very frequently employed in experimental studies $[27,28]$. In this preclinical model, the ligation of sciatic nerve by four sutures mimics the clinical situation of carpal tunnel syndrome in humans [27,29]. There have been previous studies showing that pain manifestations in this model become predominant on the 14th day [29]. Accordingly, in this study, the pain testing was done on the 14th day following surgery.

\section{Vincristine model}

Vincristine (50 $\mu \mathrm{g} / \mathrm{kg}$ i.p.) was administered for ten consecutive days to induce the development of neuropathic pain [30]. The pain testing was conducted on the 0th day (i.e., a day before starting vincristine administration) and on the 14th day of vincristine administration. Vincristine is an anticancer drug and is very frequently employed for 
the treatment of cancers [31]. Nevertheless, incidences of neuropathic pain in these patients limit the clinical utility of this anticancer drug [32]. Accordingly, vincristineinduced neuropathic pain is a widely employed preclinical model to explore new therapeutic modalities in pain management in vincristine treated patients [33].

\section{Assessment of pain-related behavior}

Three pain-related behavioral tests were performed to assess hyperalgesia and allodynia. The behavioral tests were performed in this order: the acetone drop test, the Von Frey hair test, and the pin-prick test, with a gap of 15 minutes between each test. All these tests were performed by placing the rats on an elevated cage with a wire mesh floor.

\section{1) Acetone drop test}

The characteristic feature of neuropathic pain is the development of cold allodynia against non-painful (non-noxious) cold stimuli, including acetone. Using micropipette, acetone $(100 \mu \mathrm{L})$ was applied (from below of the wired mesh) on the plantar surface of the hind paw. The duration for which the animal withdrew its paw was noted in seconds. This test was done three times with an interval of 5 minutes, and the cumulative paw withdrawal duration was noted [34].

\section{2) Von Frey hair test}

This test was also performed in the same cage in which the acetone test was performed. In this test, the development of mechanical allodynia was assessed against non-painful stimuli [35] with slight modifications [36,37]. Twenty Von Frey hair filaments (Bio-VF-M; BiosebLab, Vitrolles, France) of varying stiffness, represented in terms of bending forces $(0.008,0.02,0.04,0.07,0.16,0.4,0.6,1,1.4,2,4,6$, $8,10,15,26,60,100,180$, and $300 \mathrm{~g}$ ), were acquired. In this study, the cut off was maintained at $26 \mathrm{~g}$ [36] instead of 15.1 $\mathrm{g}$ [35]. Since the cut off was fixed at $26 \mathrm{~g}$, the $60,100,180$, and $300 \mathrm{~g}$ filaments were not used in this test. These filaments were touched to the mid-plantar region of the hind paw to induce paw withdrawal. These hairs were applied ten times in ascending order of stiffness i.e., the softest hair was applied first and hardest last. The threshold value i.e., the withdrawal threshold (in grams), was equal to the Von Frey hair stiffness, which evoked $50 \%$ paw withdrawal i.e., 5 times out of 10 trials.

\section{3) Pin-prick test (mechanical hyperalgesia)}

In the same elevated cage, the pointed end of a traumatic needle was applied to the plantar surface of the hind paw to assess mechanical hyperalgesia. The pain stimulus was applied to the plantar surface with a sufficient intensity to just evoke a paw withdrawal response, without inducing injury to the paw. This duration of paw withdrawal was recorded in seconds, and the minimum paw withdrawal duration was 0.5 seconds [38].

\section{Biochemical parameters}

After euthenizing the animals, the entire sciatic nerve (from the hip to the foot) was isolated. It was homogenized in phosphate-buffered saline, $\mathrm{pH}$ 7.4, followed by centrifugation at 2,000 g, for 15 minutes. Thereafter, the sediments were discarded and the supernatant was selected for biochemical testing.

\section{1) Quantification of $\mathrm{H}_{2} \mathrm{~S}$}

The $\mathrm{H}_{2} \mathrm{~S}$ levels were quantified in the nerve homogenate using reverse-phase chromatography. Thirty $\mu \mathrm{L}$ of the sample was added to $70 \mu \mathrm{L}$ of $100 \mathrm{mM}$ Tris-HCl buffer (pH 9.5), and thereafter, sulfide was derivatized with excess monobromobimane to form sulfide-dibimane. After 30 minutes, the reaction was stopped by adding $50 \mu \mathrm{L}$ of 200 mM 5-sulfosalicylic acid. The concentration of sulfidedibimane was measured using a fluorescence detector (excitation wavelength: $390 \mathrm{~nm}$ and emission wavelength: $475 \mathrm{~nm})$ [39].

\section{2) Quantification of CSE, CBS, orexin, and Nrf2}

The levels of CSE, orexin, and Nrf2 were quantified in the nerve homogenate using commercially available enzymelinked immunosorbent assay kits, following the instruction manual. The levels of CBS were quantified using a fluorometric assay kit in which cysteine and homocysteine were added in the homogenate to generate $\mathrm{H}_{2} \mathrm{~S}$. This was followed by the reaction of hydrogen sulfide with the azido-functional group to yield fluorescence, which was detected using an excitation wavelength of $368 \mathrm{~nm}$ and an emission wavelength of $460 \mathrm{~nm}$. Concurrently, the protein content in the homogenates was determined using FolinLowery's method [40]. All biochemical estimations were done in triplicate.

\section{Study design}

In this experimental study, fifteen groups $(\mathrm{n}=10)$ were used to meet the aim of the study. These groups included a non-treated control (not subjected to any treatment); a sham for CCI (just exposure of the sciatic nerve, no liga- 
tion); CCI (the sciatic nerve exposed and ligated); oleuropein $(10 \mathrm{mg} / \mathrm{kg})$ in CCI (treatment with oleuropein per oral [p.o.] for 14 days); oleuropein $(20 \mathrm{mg} / \mathrm{kg})$ in CCI; suvorexant $(10 \mathrm{mg} / \mathrm{kg}$ ) and oleuropein $(20 \mathrm{mg} / \mathrm{kg})$ in CCI (coadministration of suvorexant and oleuropein for 14 days); suvorexant $(20 \mathrm{mg} / \mathrm{kg})$ and oleuropein $(20 \mathrm{mg} / \mathrm{kg})$ in CCI; a sham for vincristine (administration of saline $1 \mathrm{~mL} / \mathrm{kg}$, a vehicle of vincristine for 10 days); a vincristine control (vincristine $50 \mu \mathrm{g} / \mathrm{kg}$ for ten days); oleuropein $(20 \mathrm{mg} / \mathrm{kg}$ ) in vincristine (treatment with oleuropein p.o. for 14 days); suvorexant $(20 \mathrm{mg} / \mathrm{kg})$ and oleuropein $(20 \mathrm{mg} / \mathrm{kg})$ in vincristine (treatment with suvorexant and oleuropein for 14 days); DMSO (10\% v/v) in CCI (administration of DMSO, a solvent of suvorexant in CCI-subjected rats); DMSO (10\% $\mathrm{v} / \mathrm{v}$ ) in vincristine (administration of DMSO, a solvent of suvorexant in vincristine-administered rats); oleuropein (20 mg/ $\mathrm{kg}$ ) per se (administration of oleuropein in normal rats); and suvorexant $(20 \mathrm{mg} / \mathrm{kg}$ ) per se (administration of suvorexant in normal rats) (Fig. 1).

\section{Statistical Analysis}

GraphPad Prism 7 (GraphPad Software Inc, La Jolla, CA) was employed to statistically analyze the data of this study and the data were reported as mean \pm standard deviation. The statistical analysis was done using one way analysis of variance. Thereafter, Tukey's multiple comparison test was used for post hoc analysis. A $P$ value $<0.05$ was considered to be statistically significant.

\section{RESULTS}

\section{Development of pain in CCI and vincristine models}

CCI and vincristine injection resulted in marked development of pain manifestations as observed on the 14th day, including cold allodynia assessed in the form of an

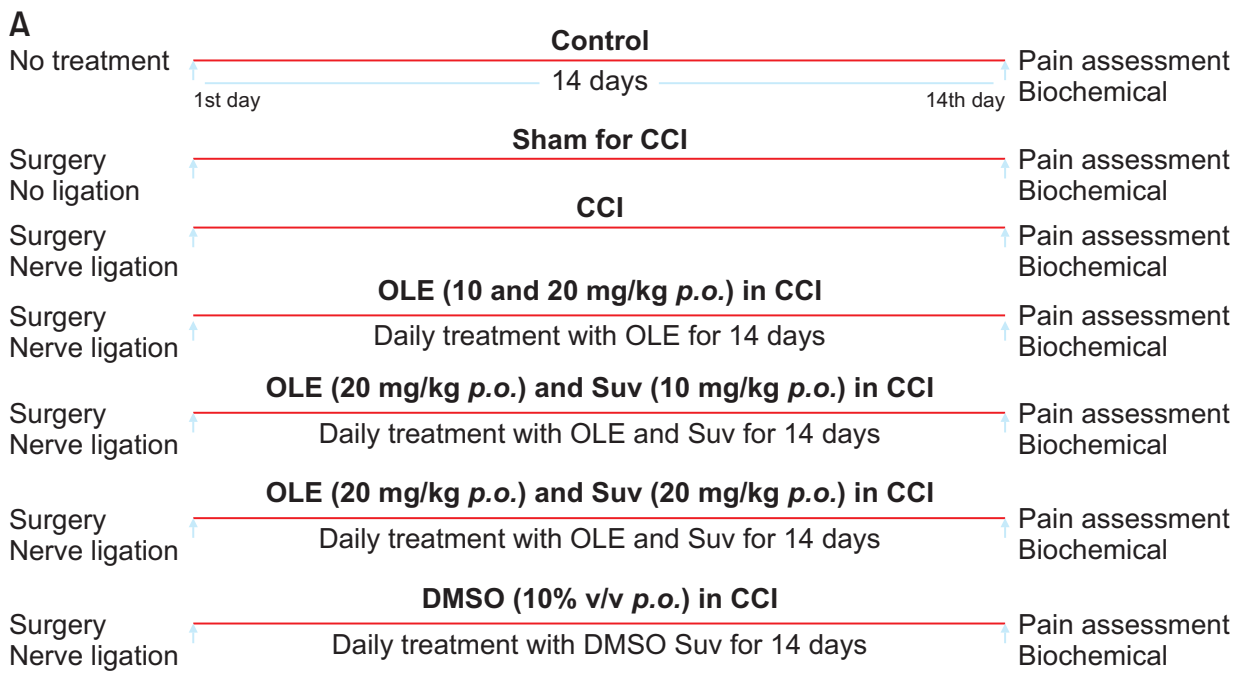

B

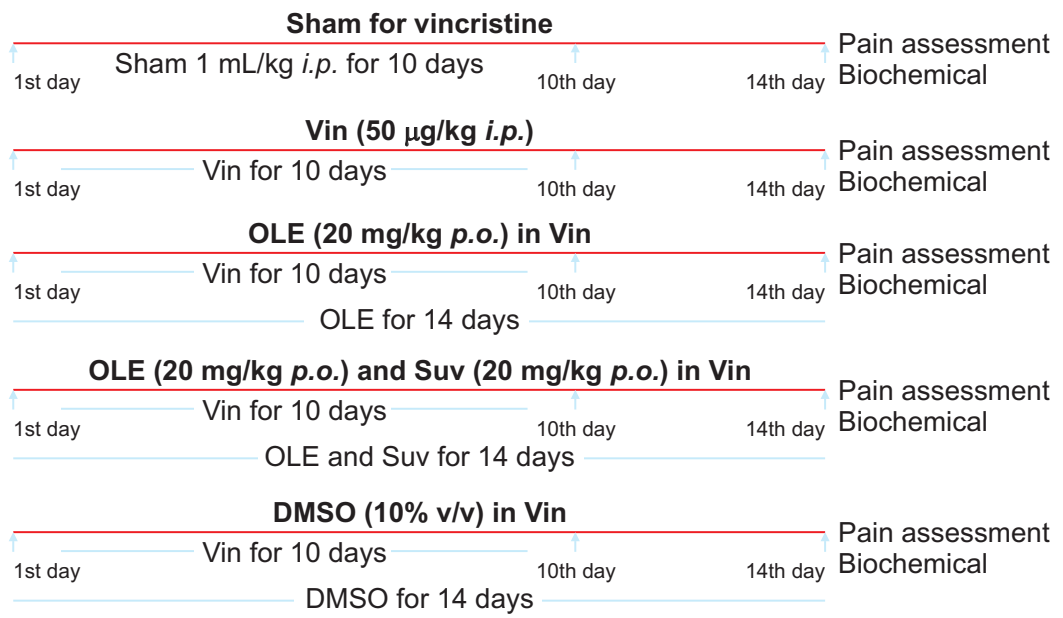

Fig. 1. Diagrammatic representation of experimental protocol in (A) chronic constriction injury (CCI) and (B) vincristine model. OLE: oleuropein, Suv: suvorexant, p.o.: per oral, i.p.: intraperitoneal, DMSO: dimethyl sulfoxide, VIN: vincristine-induced neuropathic pain. 


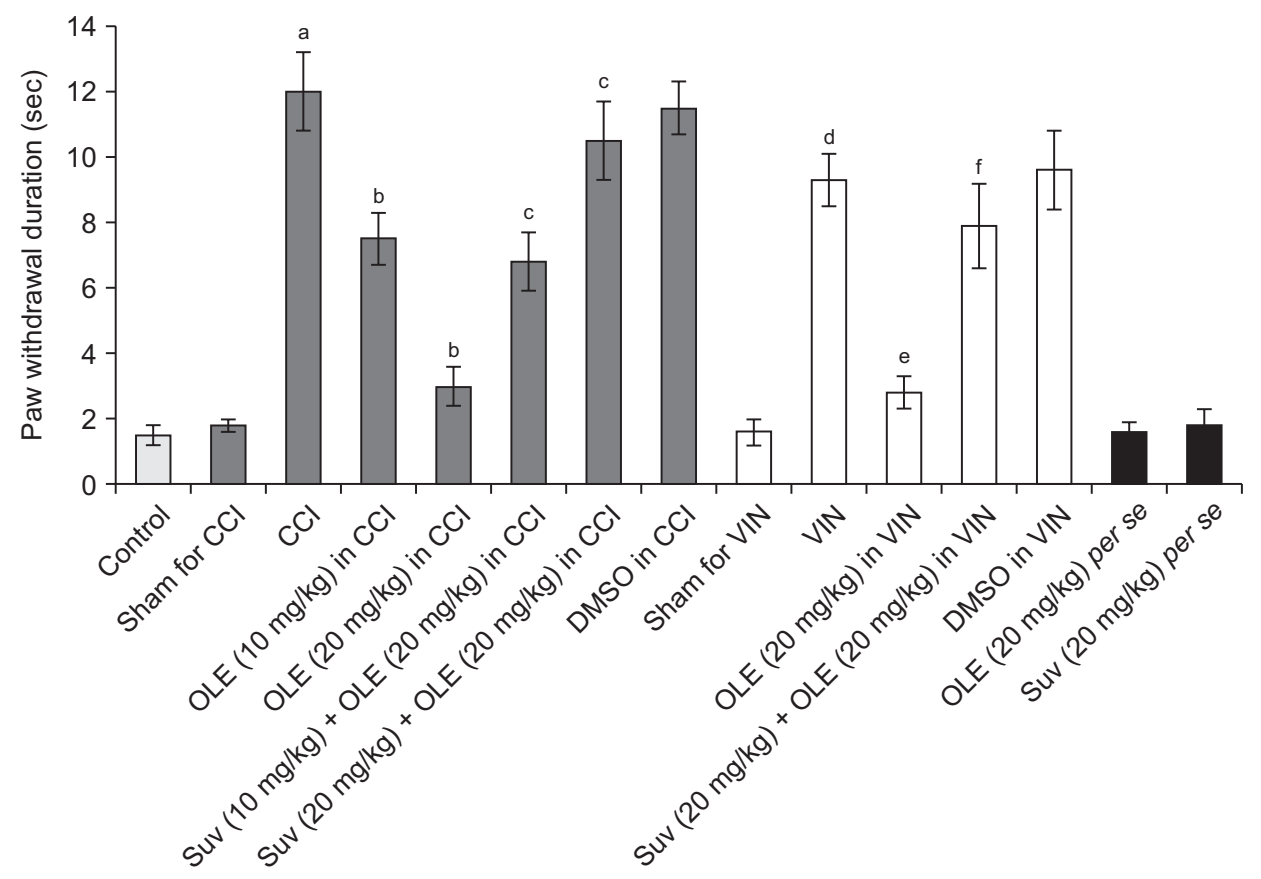

Fig. 2. Effect of different interventions on the cold allodynia in the acetone drop test in chronic constriction injury (CCl) and vincristine models. Values are in mean \pm standard deviation. $N=10, F(14,135)=342.5$. OLE: oleuropein, Suv: suvorexant, DMSO: dimethyl sulfoxide, VIN: vincristine-induced neuropathic pain. ${ }^{\mathrm{a}} P<0.05$ vs. sham for $\mathrm{CCl},{ }^{\mathrm{b}} P<0.05$ vs. $\mathrm{CCl},{ }^{\mathrm{C}} P<0.05$ vs. OLE $(20 \mathrm{mg} / \mathrm{kg})$ in CCl, ${ }^{\mathrm{d}} P<0.05$ vs. sham for VIN, ${ }^{\mathrm{e}} P<0.05 \mathrm{VIN},{ }^{\mathrm{f}} P<0.05$ OLE $(20$ $\mathrm{mg} / \mathrm{kg}$ ) in VIN.

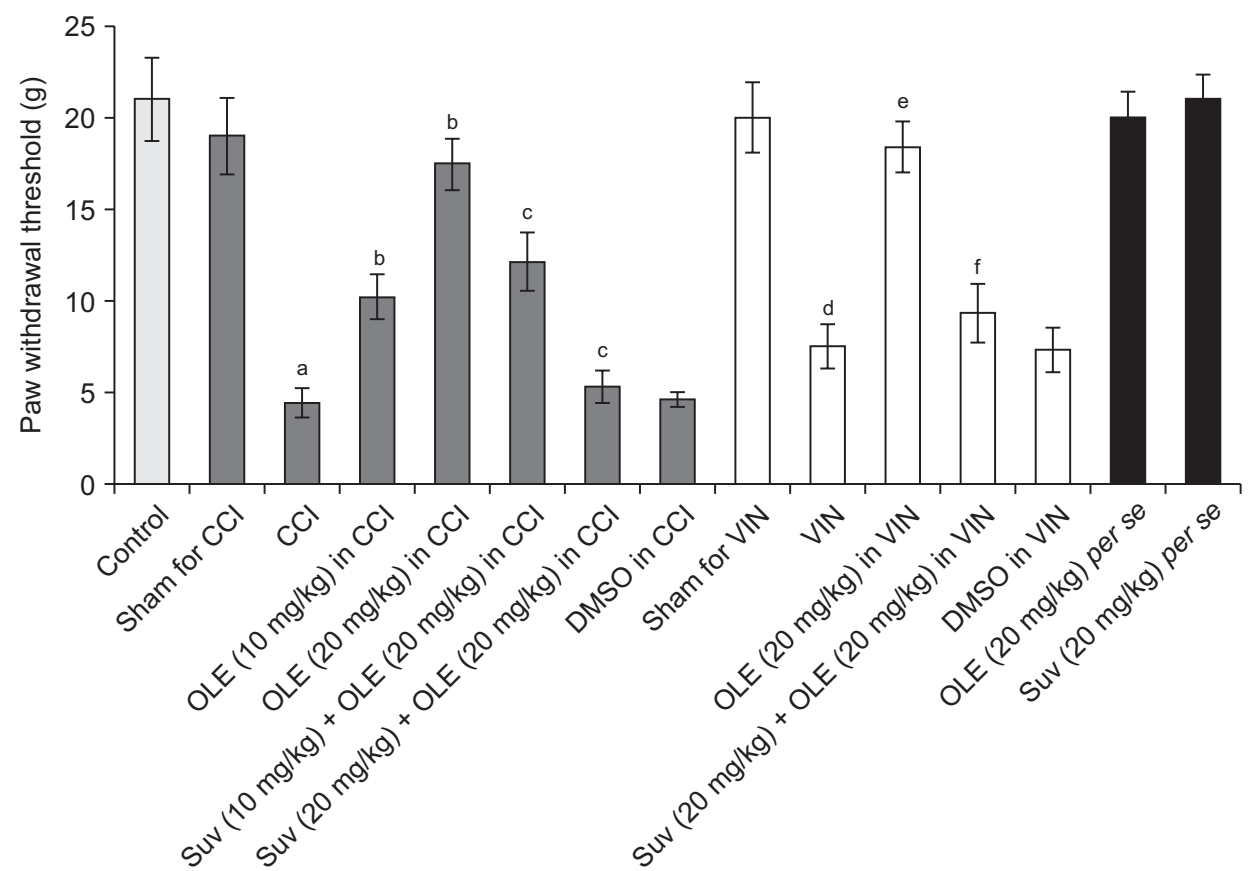

Fig. 3. Effect of different interventions on mechanical allodynia in the Von Frey filament test in chronic constriction injury (CCI) and vincristine models. Values are in mean \pm standard deviation. $N=10, F(14,135)=372.7$. OLE: oleuropein, Suv: suvorexant, DMSO: dimethyl sulfoxide, VIN: vincristineinduced neuropathic pain. ${ }^{\mathrm{a}} P<0.05$ vs. sham for CCl, ${ }^{\mathrm{b}} P<0.05$ vs. CCl, ${ }^{\mathrm{C}} P<0.05 \mathrm{vs}$. OLE $(20 \mathrm{mg} / \mathrm{kg})$ in $\mathrm{CCl},{ }^{\mathrm{d}} P<0.05$ vs. sham for VIN, ${ }^{\mathrm{e}} P<0.05 \mathrm{VIN},{ }^{\mathrm{f}} P$ $<0.05$ OLE $(20 \mathrm{mg} / \mathrm{kg})$ in VIN. 


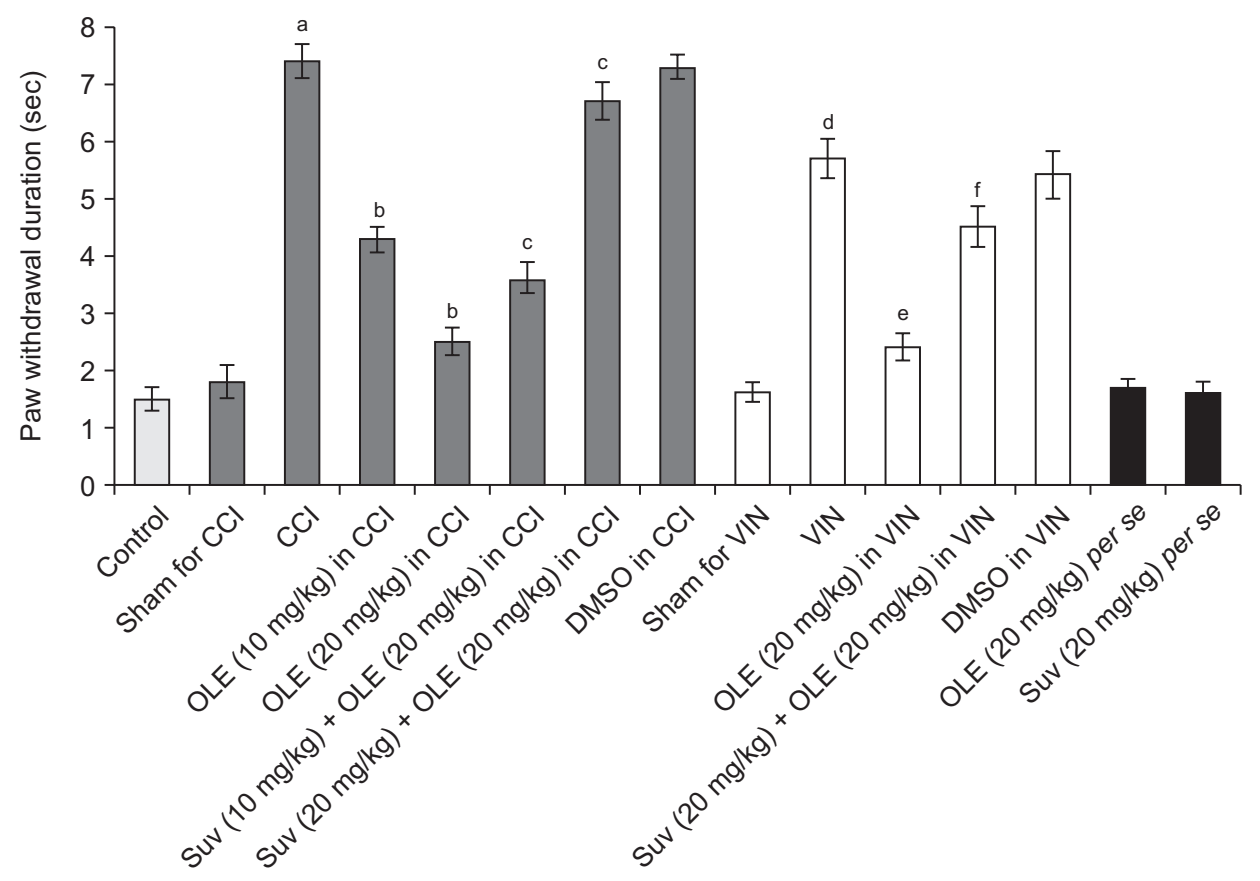

Fig. 4. Effect of different interventions on mechanical hyperalgesia in the pin-prick test in chronic constriction injury (CCl) and vincristine models. Values are in mean \pm standard deviation. $N=10, F(14,135)=313.8$. OLE: oleuropein, Suv: suvorexant, DMSO: dimethyl sulfoxide, VIN: vincristine-induced neuropathic pain. ${ }^{\mathrm{a}} P<0.05$ vs. sham for $\mathrm{CCl},{ }^{\mathrm{b}} P<0.05 \mathrm{vs}$. CCI, ${ }^{\mathrm{C}} P<0.05 \mathrm{vs}$. OLE $(20 \mathrm{mg} / \mathrm{kg})$ in CCl, ${ }^{\mathrm{d}} P<0.05$ vs. sham for VIN, ${ }^{\mathrm{e}} P<0.05 \mathrm{VIN},{ }^{\mathrm{f}} P<0.05$ OLE $(20$ $\mathrm{mg} / \mathrm{kg}$ ) in VIN.

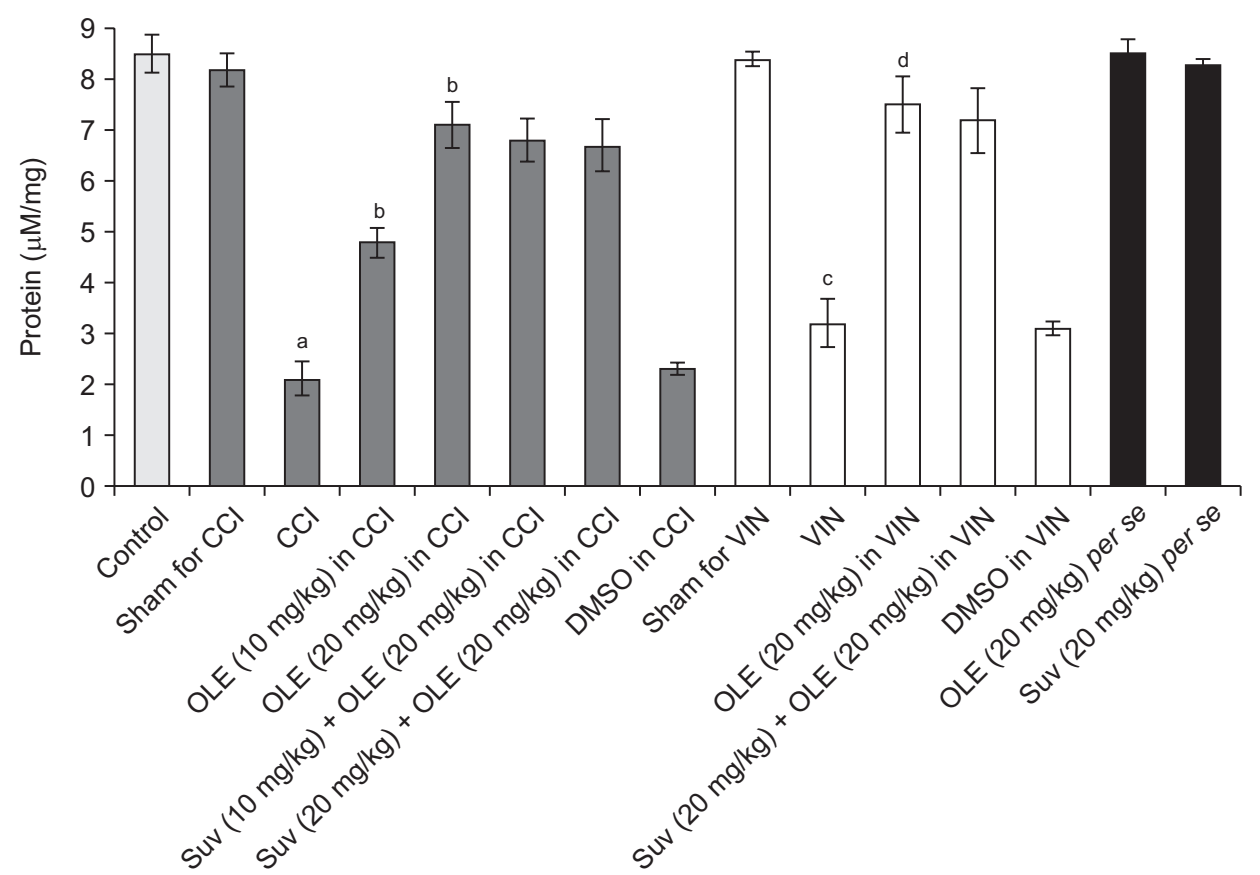

Fig. 5. Effect of different interventions on $\mathrm{H}_{2} \mathrm{~S}$ levels in the sciatic nerve in chronic constriction injury (CCl) and vincristine models. Values are in mean \pm standard deviation. $N=10, F(14,135)=140.5$. OLE: oleuropein, Suv: suvorexant, DMSO: dimethyl sulfoxide, VIN: vincristine-induced neuropathic pain. ${ }^{\mathrm{a}} P<0.05$ vs. sham for CCl, ${ }^{\mathrm{b}} P<0.05$ vs. CCI, ${ }^{\mathrm{c}} P<0.05$ vs. sham for $\mathrm{VIN},{ }^{\mathrm{d}} P<0.05 \mathrm{VIN}$. 


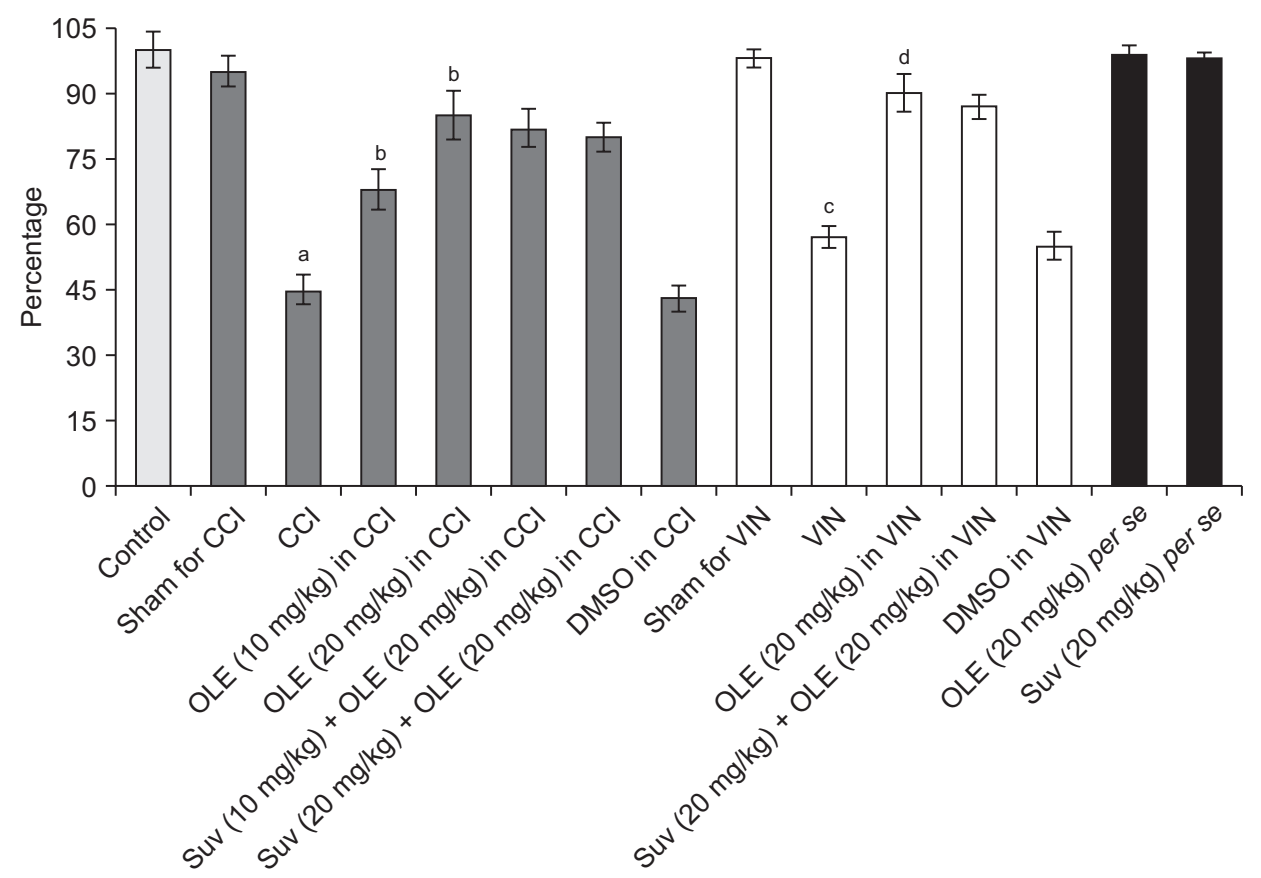

Fig. 6. Effect of different interventions on cystathionine- $\gamma-$ lyase levels in the sciatic nerve in chronic constriction injury (CCl) and vincristine models. Values are in mean \pm standard deviation. $N=10, F(14,135)=120.2$. OLE: oleuropein, Suv: suvorexant, DMSO: dimethyl sulfoxide, VIN: vincristine-induced neuropathic pain. ${ }^{\mathrm{a}} P<0.05$ vs. sham for $\mathrm{CCl},{ }^{\mathrm{b}} P<0.05$ vs. $\mathrm{CCl},{ }^{\mathrm{C}} P<0.05$ vs. sham for $\mathrm{VIN},{ }^{\mathrm{d}} P<0.05 \mathrm{VIN}$.

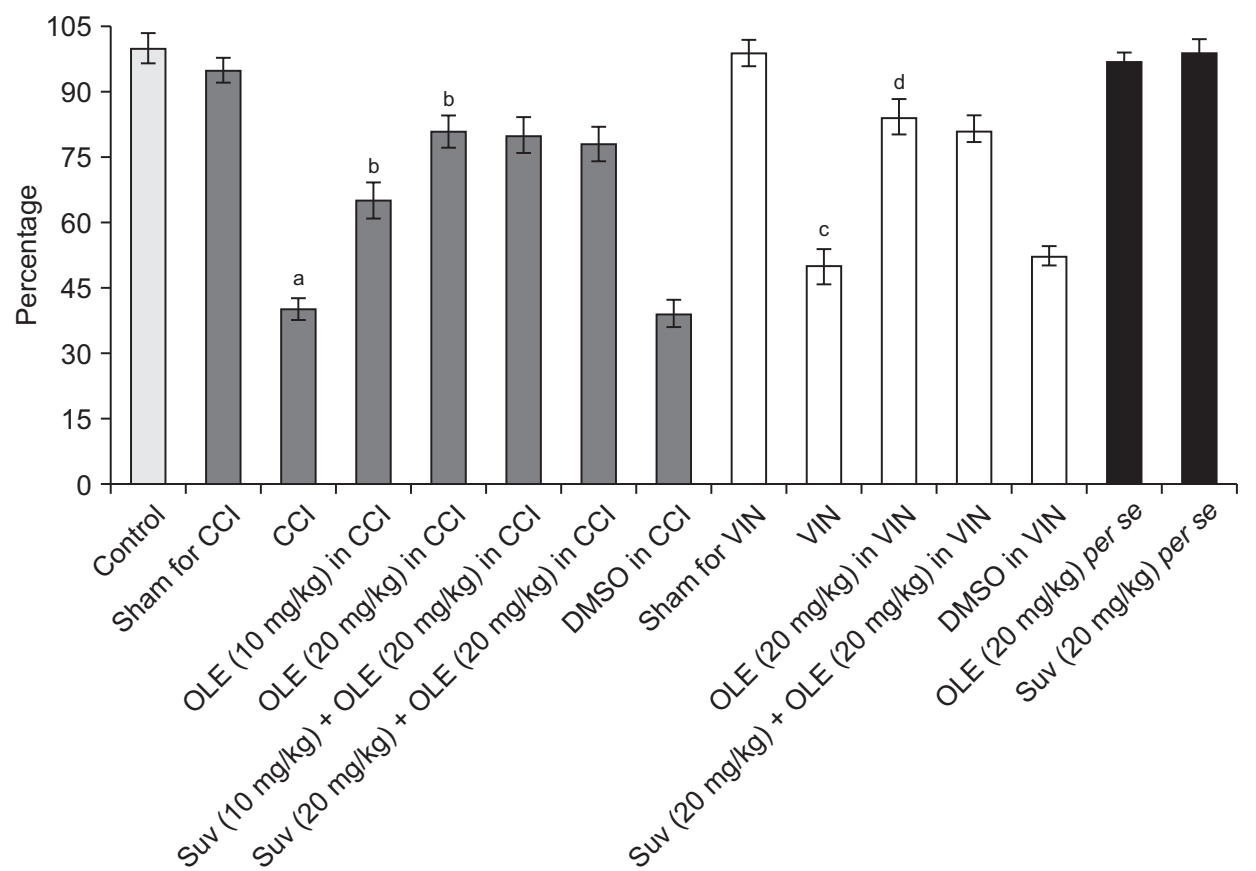

Fig. 7. Effect of different interventions in cystathionine- $\beta$-synthase levels in the sciatic nerve in chronic constriction injury (CCI) and vincristine models. Values are in mean \pm standard deviation. $N=10, F(14,135)=132.7$. OLE: oleuropein, Suv: suvorexant, DMSO: dimethyl sulfoxide, VIN: vincristineinduced neuropathic pain. ${ }^{\mathrm{a}} P<0.05$ vs. sham for $\mathrm{CCl},{ }^{\mathrm{b}} P<0.05$ vs. $\mathrm{CCl},{ }^{\mathrm{C}} P<0.05$ vs. sham for $\mathrm{VIN},{ }^{\mathrm{d}} P<0.05$ VIN. 


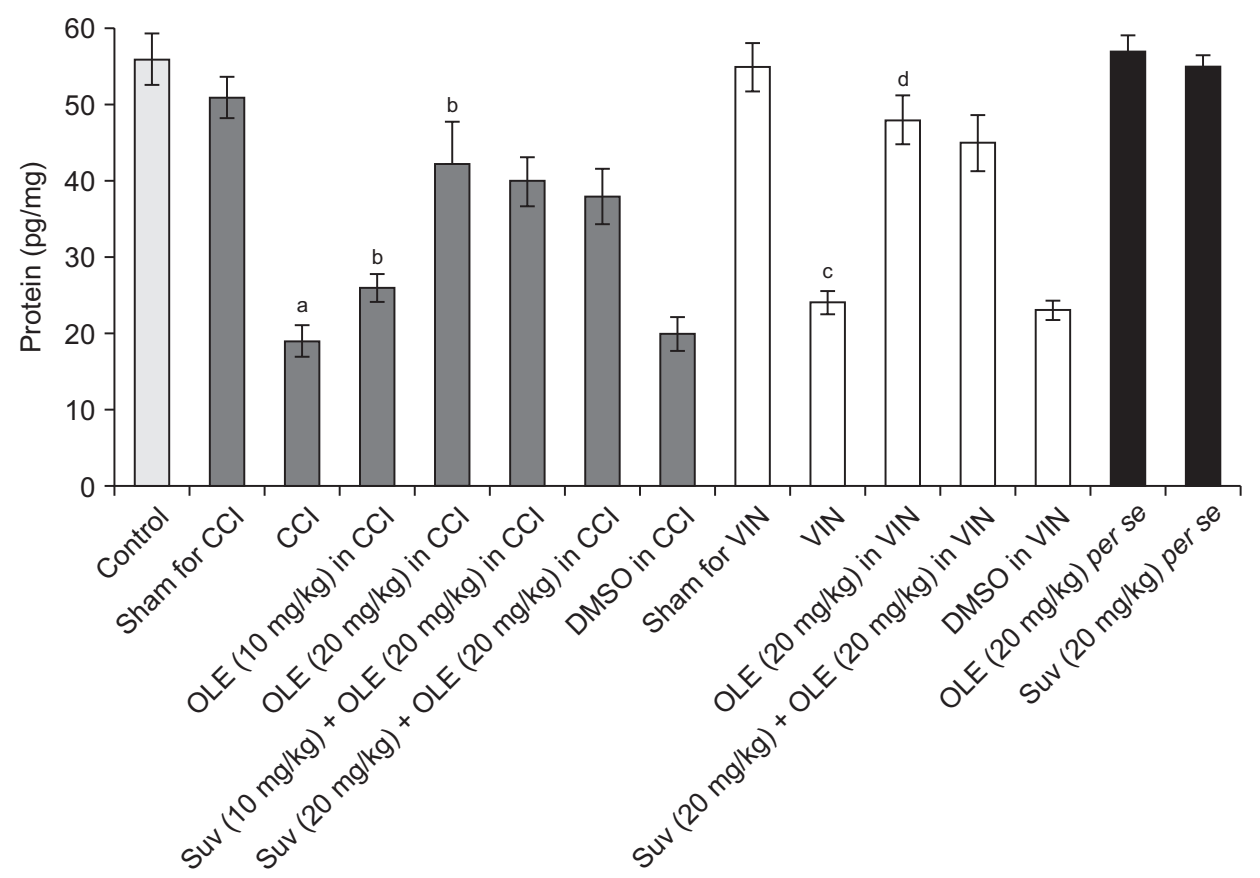

Fig. 8. Effect of different interventions on orexin levels in the sciatic nerve in chronic constriction injury (CCl) and vincristine models. Values are in mean \pm standard deviation. $N=10, F(14,135)=154.4$. OLE: oleuropein, Suv: suvorexant, DMSO: dimethyl sulfoxide, VIN: vincristine-induced neuropathic pain. ${ }^{\mathrm{a}} P<0.05$ vs. sham for $\mathrm{CCl},{ }^{\mathrm{b}} P<0.05$ vs. CCI, ${ }^{\mathrm{C}} P<0.05$ vs. sham for $\mathrm{VIN},{ }^{\mathrm{d}} P<0.05 \mathrm{VIN}$.

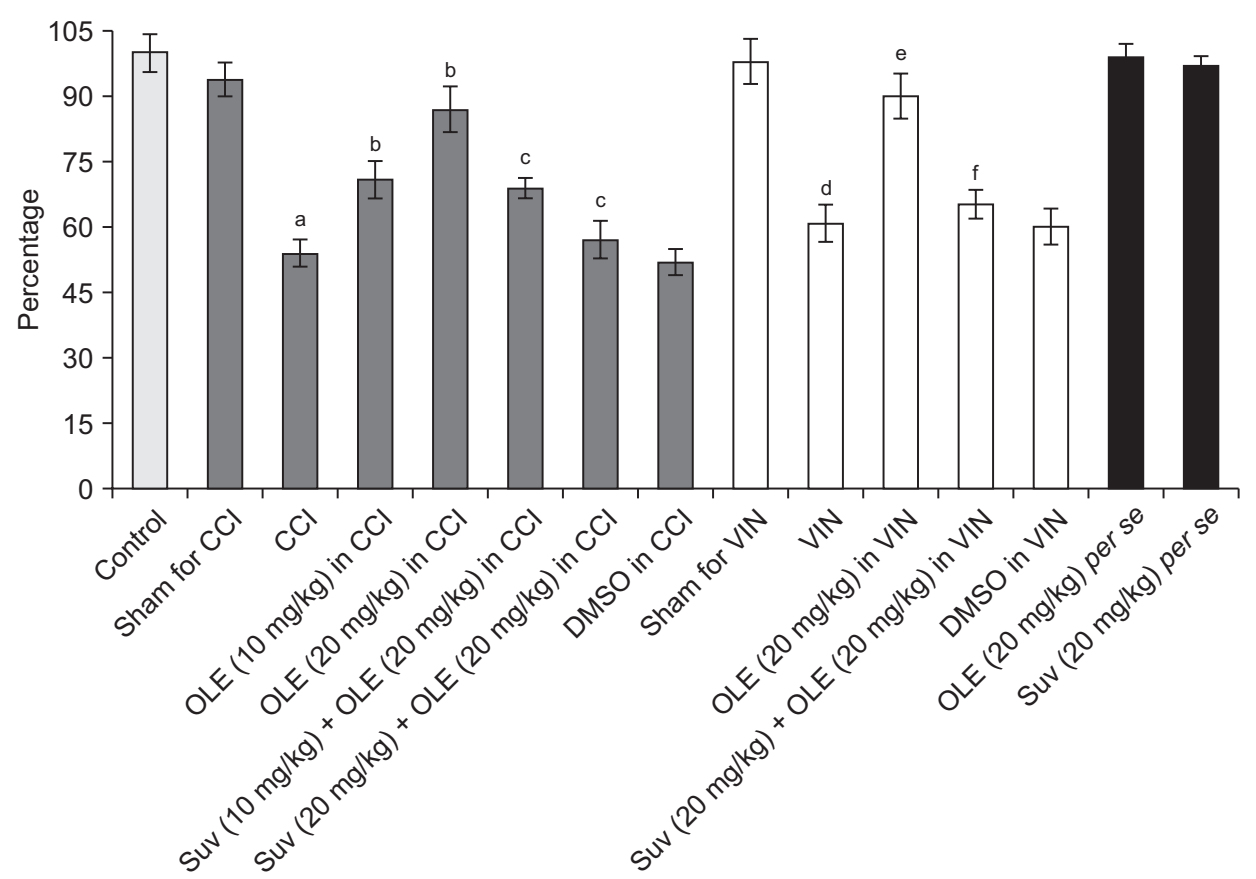

Fig. 9. Effect of different interventions on nuclear factor erythroid-2-related factor 2 levels in the sciatic nerve in chronic constriction injury (CCl) and vincristine models. Values are in mean \pm standard deviation. $N=10, F(14,135)=144.2$. OLE: oleuropein, Suv: suvorexant, DMSO: dimethyl sulfoxide, VIN: vincristine-induced neuropathic pain. ${ }^{\mathrm{a}} P<0.05$ vs. sham for CCl, ${ }^{\mathrm{b}} P<0.05 \mathrm{vs}$. CCl, ${ }^{\mathrm{C}} P<0.05 \mathrm{vs}$. OLE (20 mg/kg) in CCl, ${ }^{\mathrm{d}} P<0.05$ vs. sham for VIN, ${ }^{\mathrm{e}} P<0.05$ VIN, ${ }^{f} P<0.05$ OLE $(20 \mathrm{mg} / \mathrm{kg})$ in VIN. 
increase in paw withdrawal duration in the acetone drop test (Fig. 2). Moreover, there was a significant development of mechanical allodynia assessed in the Von Frey hair test (Fig. 3) and mechanical hyperalgesia assessed in the pinprick test (Fig. 4).

\section{Attenuation of behavioral manifestations of neuropathic pain in CCI-subjected and vincristine-administered rats with the treatment of oleuropein}

Fourteen days of treatment of CCI-subjected rats with oleuropein (10 and $20 \mathrm{mg} / \mathrm{kg}$ ) reduced the pain manifestations, including a decrease in cold allodynia (Fig. 2), mechanical allodynia (Fig. 3), and mechanical hyperalgesia (Fig. 4). Similarly, the treatment of vincristineadministered rats with oleuropein $(20 \mathrm{mg} / \mathrm{kg})$ for 14 days significantly attenuated the pain symptoms. From the results of the CCI-treated group, oleuropein $(20 \mathrm{mg} / \mathrm{kg})$ was obtained for the vincristine group.

\section{Oleuropein-mediated modulation of biochemical parameters in CCI-subjected and vincristine- administered rats}

Significant alterations in the biochemical parameters were observed in the homogenates of the sciatic nerve in both neuropathic pain models. There was a decrease in the $\mathrm{H}_{2} \mathrm{~S}$ levels along with the reduction in the CSE, CBS, and orexin. Treatment with oleuropein for 14 days led to significant elevation in $\mathrm{H}_{2} \mathrm{~S}$ (Fig. 5), CSE (Fig. 6), CBS (Fig. 7), orexin (Fig. 8), and Nrf2 (Fig. 9) in the sciatic nerve of CCIsubjected and vincristine-administered rats.

\section{Attenuation of effects of oleuropein in CCI and vincristine models in the presence of suvorexant}

Co-administration of suvorexant, an orexin receptor antagonist, significantly attenuated oleuropein-mediated pain attenuating actions in CCI-subjected and vincristineadministered rats. Suvorexant also attenuated oleuropeinmediated restoration of Nrf2 levels in the sciatic nerve (Fig. 9) in CCI-subjected and vincristine-administered rats, without influencing $\mathrm{H}_{2} \mathrm{~S}$ (Fig. 5), CSE (Fig. 6), CBS (Fig. 7), or orexin (Fig. 8).

\section{DISCUSSION}

In this experimental study, oleuropein exhibited neuropathic pain-attenuating actions and attenuated CCIinduced development of cold allodynia, mechanical allodynia, and mechanical hyperalgesia in male rats. Previous studies have reported the therapeutic potential of oleuropein in preventing cognitive decline [34], cancer, pulmonary inflammation [41], and ischemia-reperfusion injury. Moreover, there have been studies showing the usefulness of oleuropein in diabetic, inflammatory, and nociceptive pain $[5,7]$. However, this is the first experimental study suggesting the analgesic actions of oleuropein in nerve injury-induced pain. The therapeutic potential of oleuropein in neuropathic pain was further supported by the results showing the attenuation of pain manifestations in oleuropein-treated vincristine-administered male rats.

Along with the development of neuropathic pain, there were significant biochemical changes in the sciatic nerve, including a decrease in the levels of $\mathrm{H}_{2} \mathrm{~S}$, CSE, and CBS. CSE and CBS synthesize hydrogen sulfide [42], which plays an important role in the pathophysiology of neuropathic pain $[13,43]$. In this current experimental investigation, treatment with oleuropein restored the levels of $\mathrm{H}_{2} \mathrm{~S}$, CSE, and CBS in the sciatic nerve in CCI-induced and vincristine-administered rats. It suggests that oleuropeinmediated therapeutic effects in neuropathic pain may be due to an increase in the expression of CSE and CBS, along with the increase in $\mathrm{H}_{2} \mathrm{~S}$. In this present study, the biochemical alterations were measured in the sciatic nerve (in the periphery), as nerve injury-induced peripheral sensitization is very important in inducing long-lasting pain in neuropathy [44-47]. Therefore, it may be proposed that oleuropein-mediated normalization of the biochemical milieu in the injured nerve may prevent peripheral nerve sensitization in the form of a decrease in abnormal, spontaneous, and ectopic activity. However, there is also a critical role for central changes (neurons of the dorsal horn and others) in inducing neuropathic pain in nerve injurysubjected rats [48]. Accordingly, there is a need for more experimental studies to explore the role of central changes in oleuropein-mediated analgesic actions.

In this investigation, the expression of orexin and Nrf2 were also attenuated in the sciatic nerve in CCI and vincristine models. However, oleuropein restored the expression of orexin and Nrf2 in neuropathic pain models, suggesting that restoration of orexin and Nrf2 may be critical in the pain attenuating action of oleuropein. A decrease in the expression of orexin [16] and Nrf2 [12] is critical in the pathogenesis of neuropathic pain. Oleuropein increases the expression of Nrf2 to produce beneficial effects in different diseases [49]. However, this is the first study showing that oleuropein may produce analgesic actions by increasing the expression of orexin and Nrf2. The key role of orexin in oleuropein-mediated beneficial effects in neuropathic pain models was supported by this investigation, showing that co-administration of suvorexant, an orexin 
receptor antagonist [50], attenuated the analgesic actions of oleuropein in CCI and vincristine models.

Administration of suvorexant also abolished the oleuropein-mediated increase in the expression of Nrf2, suggesting that oleuropein may increase the expression of orexin, which may subsequently increase the expression of Nrf2. Nrf2 has been reported as the downstream mediator of orexin [41]. However, administration of an orexin receptor blocker (suvorexant) did not attenuate the levels of orexin in oleuropein-treated rats. Generally, the receptor blockers attenuate the biological actions of agonists by preventing their binding to receptor sites, rather than attenuating their biosynthesis. Therefore, it is possible that suvorexant may have attenuated the actions of orexin by preventing its binding to orexin receptors. Nevertheless, more studies may be done to precisely elucidate the reason for the nonmodulation of orexin levels in the presence of suvorexant. Moreover, suvorexant did not alter the expression of $\mathrm{H}_{2} \mathrm{~S}$, CSE, and CBS. In other words, the blockade of the actions of the orexin receptors did not affect $\mathrm{H}_{2} \mathrm{~S}$, CSE, and CBS, suggesting that orexin is the downstream mediator of $\mathrm{H}_{2} \mathrm{~S}$. Accordingly, it may be hypothesized that oleuropein may up-regulate the expression of CSE and CBS to increase the $\mathrm{H}_{2} \mathrm{~S}$ levels, which may be followed by an increase in the expression of orexin and Nrf2 to ameliorate neuropathic pain in rats.

Despite the increase in levels of $\mathrm{H}_{2} \mathrm{~S}$, the lack of any beneficial effects from oleuropein in suvorexant-treated rats also suggests that the rise in the peripheral $\mathrm{H}_{2} \mathrm{~S}$ levels may not be contributing in a significant manner to attenuating pain manifestations. In other words, oleuropein-mediated modulation of orexin and Nrf2 may be more important mechanisms in comparison to the increase in $\mathrm{H}_{2} \mathrm{~S}$ levels. However, the previous studies showing the role of $\mathrm{H}_{2} \mathrm{~S}$ in pain modulation and the present study results showing an increase in the expression of $\mathrm{H}_{2} \mathrm{~S}$ biosynthetic enzymes, along with an increase in the $\mathrm{H}_{2} \mathrm{~S}$ levels in oleuropeintreated rats, also suggest the important role of $\mathrm{H}_{2} \mathrm{~S}$ in oleuropein-mediated pain attenuating actions.

It may be concluded that oleuropein has therapeutic potential to attenuate the pain manifestations in CCI and vincristine-induced neuropathic pain, possibly by restoring the CSE, CBS, and $\mathrm{H}_{2} \mathrm{~S}$, which may subsequently increase the expression of orexin and Nrf2 to ameliorate behavioral manifestations of pain. Future experimental investigations are needed to delineate the precise role of $\mathrm{H}_{2} \mathrm{~S}$ in oleuropein-mediated pain attenuating actions by employing exogenous $\mathrm{H}_{2} \mathrm{~S}$ donors and selective pharmacological inhibitors of CSE and CBS. Moreover, further studies are also required to clearly delineate the interrelationship between $\mathrm{H}_{2} \mathrm{~S}$, Nrf2, and orexin in oleuropeinmediated neuropathic pain attenuating actions in rats.

\section{CONFLICT OF INTEREST}

No potential conflict of interest relevant to this article was reported.

\section{FUNDING}

No funding to declare.

\section{ORCID}

Huayong Chen, https://orcid.org/0000-0002-2383-0539

Dandan Ma, https://orcid.org/0000-0003-0320-5362

Huapeng Zhang, https://orcid.org/0000-0002-8729-6523

Yanhong Tang, https://orcid.org/0000-0002-4533-3824

Jun Wang, https://orcid.org/0000-0003-2475-6585

Renhu Li, https://orcid.org/0000-0003-3005-1389

Wen Wen, https://orcid.org/0000-0001-6018-054X

Yi Zhang, https://orcid.org/0000-0002-6156-1894

\section{REFERENCES}

1. Zilliox LA. Neuropathic Pain. Continuum (Minneap Minn) 2017; 23(2, Selected Topics in Outpatient Neurology): 512-32.

2. Badr AM, Attia HA, Al-Rasheed N. Oleuropein reverses repeated corticosterone-induced depressive-like behavior in mice: evidence of modulating effect on biogenic amines. Sci Rep 2020; 10: 3336.

3. Mohammad-Beigi H, Aliakbari F, Sahin C, Lomax C, Tawfike A, Schafer NP, et al. Oleuropein derivatives from olive fruit extracts reduce $\alpha$-synuclein fibrillation and oligomer toxicity. J Biol Chem 2019; 294: 4215-32.

4. Fki I, Sayadi S, Mahmoudi A, Daoued I, Marrekchi R, Ghorbel H. Comparative study on beneficial effects of hydroxytyrosol- and oleuropein-rich olive leaf extracts on high-fat diet-induced lipid metabolism disturbance and liver injury in rats. Biomed Res Int 2020; 2020: 1315202.

5. Czerwińska ME, Gąsińska E, Leśniak A, Krawczyk P, Kiss AK, Naruszewicz M, et al. Inhibitory effect of Ligustrum vulgare leaf extract on the development of neuropathic pain in a streptozotocin-induced rat model of diabetes. Phytomedicine 2018; 49: 75-82.

6. Mao X, Xia B, Zheng M, Zhou Z. Assessment of the antiinflammatory, analgesic and sedative effects of oleuropein from Olea europaea L. Cell Mol Biol (Noisy-le-grand) 2019; 65: $52-5$.

7. Zare L, Esmaeili-Mahani S, Abbasnejad M, Rasoulian B, Sheibani V, Sahraei H, et al. Oleuropein, chief constituent of olive leaf extract, prevents the development of morphine 
antinociceptive tolerance through inhibition of morphineinduced L-type calcium channel overexpression. Phytother Res 2012; 26: 1731-7.

8. Linden DR. Hydrogen sulfide signaling in the gastrointestinal tract. Antioxid Redox Signal 2014; 20: 818-30.

9. $\mathrm{Wu} \mathrm{D}, \mathrm{Hu} \mathrm{Q}$, Zhu D. An update on hydrogen sulfide and nitric oxide interactions in the cardiovascular system. Oxid Med Cell Longev 2018; 2018: 4579140.

10. Sheibani L, Lechuga TJ, Zhang H, Hameed A, Wing DA, Kumar S, et al. Augmented H2S production via cystathioninebeta-synthase upregulation plays a role in pregnancyassociated uterine vasodilation. Biol Reprod 2017; 96: 664-72.

11. Lin JQ, Luo HQ, Lin CZ, Chen JZ, Lin XZ. Sodium hydrosulfide relieves neuropathic pain in chronic constriction injured rats. Evid Based Complement Alternat Med 2014; 2014: 514898.

12. Chen H, Xie K, Chen Y, Wang Y, Wang Y, Lian N, et al. Nrf2/ HO-1 signaling pathway participated in the protection of hydrogen sulfide on neuropathic pain in rats. Int Immunopharmacol 2019; 75: 105746.

13. Lucarini E, Micheli L, Trallori E, Citi V, Martelli A, Testai L, et al. Effect of glucoraphanin and sulforaphane against chemotherapy-induced neuropathic pain: Kv7 potassium channels modulation by H2 S release in vivo. Phytother Res 2018; 32: 2226-34

14. Mieda M. The roles of orexins in sleep/wake regulation. Neurosci Res 2017; 118: 56-65.

15. Suyama H, Kawamoto M, Shiraishi S, Gaus S, Kajiyama S, Yuge O. Analgesic effect of intrathecal administration of orexin on neuropathic pain in rats. In Vivo 2004; 18: 119-23.

16. Toyama S, Shimoyama N, Shimoyama M. The analgesic effect of orexin-A in a murine model of chemotherapy-induced neuropathic pain. Neuropeptides 2017; 61: 95-100.

17. Kajiyama S, Kawamoto M, Shiraishi S, Gaus S, Matsunaga A, Suyama H, et al. Spinal orexin-1 receptors mediate antihyperalgesic effects of intrathecally-administered orexins in diabetic neuropathic pain model rats. Brain Res 2005; 1044: 76-86.

18. Ma Q. Role of nrf2 in oxidative stress and toxicity. Annu Rev Pharmacol Toxicol 2013; 53: 401-26.

19. Yetik-Anacak G, Sevin G, Ozzayım O, Dereli MV, Ahmed A. Hydrogen sulfide: a novel mechanism for the vascular protection by resveratrol under oxidative stress in mouse aorta. Vascul Pharmacol 2016; 87: 76-82.

20. Wang Z, Yan Y, Wang Y, Tong F. The interaction between CSE/ $\mathrm{H}_{2} \mathrm{~S}$ and the iNOS/NO-mediated resveratrol/poly(ethylene glycol)-poly(phenylalanine) complex alleviates intestinal ischemia/reperfusion injuries in diabetic rats. Biomed Pharmacother 2019; 112: 108736.

21. Vitalone A, Di Sotto A, Mammola CL, Heyn R, Miglietta S, Mariani $\mathrm{P}$, et al. Phytochemical analysis and effects on ingestive behaviour of a Caralluma fimbriata extract. Food
Chem Toxicol 2017; 108(Pt A): 63-73.

22. Guo C, Bi J, Li X, Lyu J, Liu X, Wu X, et al. Immunomodulation effects of polyphenols from thinned peach treated by different drying methods on RAW264.7 cells through the NF$\kappa B$ and Nrf2 pathways. Food Chem 2021; 340: 127931.

23. Castejon ML, Sánchez-Hidalgo M, Aparicio-Soto M, Montoya T, Martín-LaCave I, Fernández-Bolaños JG, et al. Dietary oleuropein and its new acyl-derivate attenuate murine lupus nephritis through HO-1/Nrf2 activation and suppressing JAK/STAT, NF- $\kappa$ B, MAPK and NLRP3 inflammasome signaling pathways. J Nutr Biochem 2019; 74: 108229.

24. Sanchez-Alavez M, Benedict J, Wills DN, Ehlers CL. Effect of suvorexant on event-related oscillations and EEG sleep in rats exposed to chronic intermittent ethanol vapor and protracted withdrawal. Sleep 2019; 42: zsz020.

25. Janahmadi Z, Nekooeian AA, Moaref AR, Emamghoreishi M. Oleuropein attenuates the progression of heart failure in rats by antioxidant and antiinflammatory effects. Naunyn Schmiedebergs Arch Pharmacol 2017; 390: 245-52.

26. Bennett GJ, Xie YK. A peripheral mononeuropathy in rat that produces disorders of pain sensation like those seen in man. Pain 1988; 33: 87-107.

27. Ma L, Liu H, Chen G, Chen M, Wang L, Zhang X, et al. Sulfasalazine attenuates chronic constriction injury-induced neuroinflammation and mechanical hypersensitivity in rats. Neurosci Lett 2018; 683: 174-80.

28. Shibayama M, Kuniyoshi K, Suzuki T, Yamauchi K, Ohtori S, Takahashi K. The effects of locally injected triamcinolone on entrapment neuropathy in a rat chronic constriction injury model. J Hand Surg Am 2014; 39: 1714-21.

29. Khangura RK, Bali A, Kaur G, Singh N, Jaggi AS. Neuropathic pain attenuating effects of perampanel in an experimental model of chronic constriction injury in rats. Biomed Pharmacother 2017; 94: 557-63.

30. Siau C, Bennett GJ. Dysregulation of cellular calcium homeostasis in chemotherapy-evoked painful peripheral neuropathy. Anesth Analg 2006; 102: 1485-90.

31. Selawry OS, Hananian J. Vincristine treatment of cancer in children. JAMA 1963; 183: 741-6.

32. Gilchrist L, Tanner L. Gait patterns in children with cancer and vincristine neuropathy. Pediatr Phys Ther 2016; 28: 1622.

33. Vashistha B, Sharma A, Jain V. Ameliorative potential of ferulic acid in vincristine-induced painful neuropathy in rats: an evidence of behavioral and biochemical examination. Nutr Neurosci 2017; 20: 60-70.

34. Choi Y, Yoon YW, Na HS, Kim SH, Chung JM. Behavioral signs of ongoing pain and cold allodynia in a rat model of neuropathic pain. Pain 1994; 59: 369-76.

35. Chaplan SR, Bach FW, Pogrel JW, Chung JM, Yaksh TL. Quantitative assessment of tactile allodynia in the rat paw. J Neurosci Methods 1994; 53: 55-63. 
36. Wang G, Yang Y, Wang C, Huang J, Wang X, Liu Y, et al. Exploring the role and mechanisms of diallyl trisulfide and diallyl disulfide in chronic constriction-induced neuropathic pain in rats. Korean J Pain 2020; 33: 216-25.

37. Xie T, Zhang J, Kang Z, Liu F, Lin Z. miR-101 down-regulates mTOR expression and attenuates neuropathic pain in chronic constriction injury rat models. Neurosci Res 2020; 158: 30 6.

38. Yamamoto S, Suzuki Y, Ono H, Kume K, Ohsawa M. N- and L-type calcium channels blocker cilnidipine ameliorates neuropathic pain. Eur J Pharmacol 2016; 793: 66-75.

39. Shen X, Chakraborty S, Dugas TR, Kevil CG. Hydrogen sulfide measurement using sulfide dibimane: critical evaluation with electrospray ion trap mass spectrometry. Nitric Oxide 2014; 41: 97-104

40. Lowry OH, Rosebrough NJ, Farr AL, Randall RJ. Protein measurement with the Folin phenol reagent. J Biol Chem 1951; 193: 265-75.

41. Kim YH, Choi YJ, Kang MK, Lee EJ, Kim DY, Oh H, et al. Oleuropein curtails pulmonary inflammation and tissue destruction in models of experimental asthma and emphysema. J Agric Food Chem 2018; 66: 7643-54.

42. Lechuga TJ, Qi QR, Kim T, Magness RR, Chen DB. E2 $\beta$ stimulates ovine uterine artery endothelial cell $\mathrm{H} 2 \mathrm{~S}$ production in vitro by estrogen receptor-dependent upregulation of cystathionine $\beta$-synthase and cystathionine $\gamma$-lyase expression $\dagger$. Biol Reprod 2019; 100: 514-22.

43. Di Cesare Mannelli L, Lucarini E, Micheli L, Mosca I, Ambrosino $\mathrm{P}$, Soldovieri MV, et al. Effects of natural and synthetic isothiocyanate-based H2S-releasers against chemotherapyinduced neuropathic pain: role of $\mathrm{Kv} 7$ potassium channels. Neuropharmacology 2017; 121: 49-59.

44. Wu G, Ringkamp M, Hartke TV, Murinson BB, Campbell JN, Griffin JW, et al. Early onset of spontaneous activity in uninjured C-fiber nociceptors after injury to neighboring nerve fibers. J Neurosci 2001; 21: RC140.

45. Djouhri L, Koutsikou S, Fang X, McMullan S, Lawson SN. Spontaneous pain, both neuropathic and inflammatory, is related to frequency of spontaneous firing in intact C-fiber nociceptors. J Neurosci 2006; 26: 1281-92.

46. Meacham K, Shepherd A, Mohapatra DP, Haroutounian S. Neuropathic pain: central vs. peripheral mechanisms. Curr Pain Headache Rep 2017; 21: 28.

47. Razavi BM, Hosseinzadeh H. A review of the role of orexin system in pain modulation. Biomed Pharmacother 2017; 90: 187-93.

48. Sorge RE, Mapplebeck JC, Rosen S, Beggs S, Taves S, Alexander JK, et al. Different immune cells mediate mechanical pain hypersensitivity in male and female mice. Nat Neurosci 2015; 18: 1081-3.

49. Castejón ML, Rosillo MÁ, Montoya T, González-Benjumea A, Fernández-Bolaños JG, Alarcón-de-la-Lastra C. Oleuropein down-regulated IL-1 $\beta$-induced inflammation and oxidative stress in human synovial fibroblast cell line SW982. Food Funct 2017; 8: 1890-8.

50. Dubey AK, Handu SS, Mediratta PK. Suvorexant: the first orexin receptor antagonist to treat insomnia. J Pharmacol Pharmacother 2015; 6: 118-21. 